Musées, Patrimoine et Culture scientifiques et techniques

$121 \mid 2009$

janvier - février 2009

\title{
Une nouvelle Cité des Enfants
}

\section{Marie-Pierre Lahalle}

URL : http://journals.openedition.org/ocim/274

DOI : $10.4000 /$ ocim. 274

ISSN : 2108-646X

\section{Éditeur}

OCIM

Édition imprimée

Date de publication : 1 janvier 2009

Pagination : 28-36

ISSN : 0994-1908

Référence électronique

Marie-Pierre Lahalle, "Une nouvelle Cité des Enfants », La Lettre de I'OCIM [En ligne], 121 | 2009, mis en ligne le 19 janvier 2011, consulté le 19 avril 2019. URL : http://journals.openedition.org/ocim/274 ; DOI : 10.4000/ocim.274 


\section{Une nouvelle Cité des Enfants}

Marie-Pierre Lahalle *

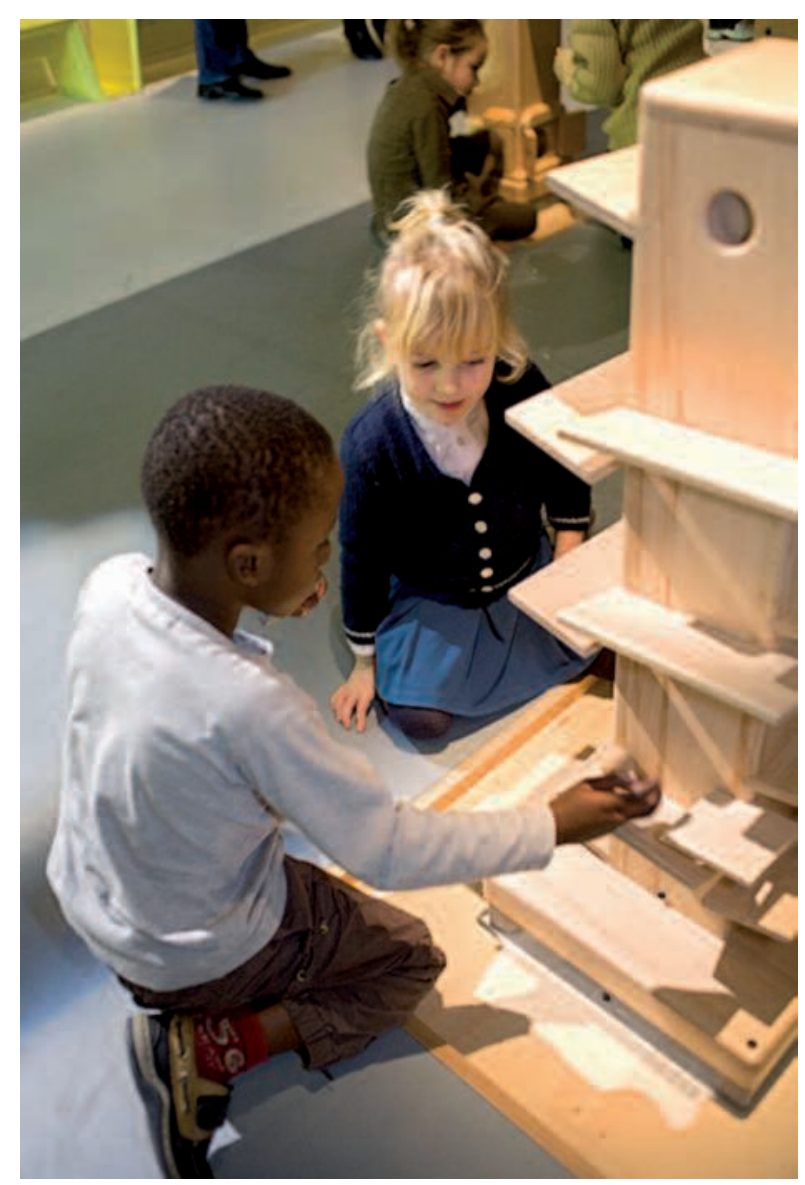

La tour en bois de la nouvelle Cité des Enfants (- CSI/Sophie Chivet

* Marie-Pierre Lahalle est responsable de la rénovation de la Cité des Enfants à la Cité des Sciences et de l'Industrie téléphone + 33140058315 lahalle@cite-sciences.fr
La Cité des Sciences et de l'Industrie a engagé depuis 2005 un ambitieux projet de renouvellement de la Cité des Enfants. L'auteur met ici en lumière les réflexions et principes qui ont guidé cette rénovation dont la première étape a vu le jour en 2008 avec l'ouverture de la Cité des Enfants pour les 2-7 ans et qui s'achèvera en avril 2009 avec celle pour les 5-12 ans.

\section{La Cité des Enfants}

La Cité des Enfants est l'une des offres majeures de la Cité des Sciences et de l'Industrie, attirant près de la moitié de notre public mais occupant seulement 19 \% de la surface de la Cité. Créée en 1992 dans la continuité de l'Inventorium (espace enfants de la Cité dès 1986), la Cité des Enfants est un espace permanent de découvertes actives pour les enfants. Sa vocation est de contribuer à leur développement et de les initier aux sciences en leur proposant la visite mémorable d'espaces d'exposition spécialement conçus à leur intention et à leur mesure.

La Cité des Enfants de 1992 occupait une surface de $2600 \mathrm{~m}^{2}$ environ répartie en deux espaces pour des enfants d'âges et de maturité différents :

- un espace d'initiation aux sciences et aux techniques pour les 5 à 12 ans (environ $1800 \mathrm{~m}^{2}$ et une centaine d'éléments d'exposition);

- un espace intitulé « Première découverte » pour les 3 à 5 ans $\left(825 \mathrm{~m}^{2}\right.$ et une cinquantaine d'éléments d'exposition), qui fut créé quelques mois après l'espace des grands, comme un espace complémentaire pour les plus jeunes. 
La conception de la Cité des Enfants s'inscrivait dans la mouvance de la muséographie anglo-saxonne des années 1980, dite Hands On : des espaces d'exposition très ouverts proposant une série de jeux d'éveil pour les enfants basés essentiellement sur l'action et l'interactivité. L'espace pour les 5-12 ans était structuré en 4 grands thèmes balayant les principaux champs de la science et de la technique : Machines et mécanismes, Enquêtes sur le vivant, Toi et les autres, Techniques pour communiquer. La partie 3-5 ans, quant à elle, était plus petite (moins de la moitié de la surface des 5-12 ans) et sa structuration thématique moins explicite car les enfants plus jeunes sont non lecteurs. L'objectif de cet espace dédié aux touts petits était d'enrichir leur perception d'eux-mêmes et du monde qui les entoure, à travers des jeux favorisant l'expérimentation, la manipulation tout en s'amusant.

Nous souhaitions aussi que les activités proposées soient originales, étonnantes, qu'elles différent radicalement de ce que l'enfant peut trouver chez lui ou à l'école. C'est pourquoi de nombreux jeux étaient basés sur l'informatique, l'univers multimédia étant alors très peu répandu dans les familles et inexistant dans des écoles.

Un autre principe fédérateur de la Cité des Enfants était la co-éducation. Bien que centrée sur l'enfant, la Cité des Enfants attachait une grande importance aux relations et aux échanges entre enfants et parents ou accompagnateurs pendant la visite. La Cité des Enfants n'est pas un jardin public ni une « garderie». On y vient pour passer un moment amusant et enrichissant, en famille ou en classe. C'est pourquoi les enfants, même de 10-12 ans, doivent être accompagnés d'un adulte pour visiter cet espace.

\section{La rénovation}

La nouvelle configuration s'inscrit dans une totale continuité avec la Cité des Enfants d'origine: ses ambitions et ses objectifs restent les mêmes, ses grands principes fédérateurs également et son fonctionnement (visite par séance et avec adulte, accueil du public, animations) tout autant.

\section{Pourquoi cette rénovation?}

En 2005, il nous a pourtant semblé nécessaire de rénover la Cité des Enfants, après 15 ans de succès et 7,5 millions de visiteurs, notamment pour :

- augmenter les surfaces d'exposition et satisfaire davantage de visiteurs, en particulier pour l'espace des petits le plus souvent saturé. Ainsi, la nouvelle
Cité des Enfants s'agrandit de manière significative pour occuper une superficie totale de $5000 \mathrm{~m}^{2}$ (hall d'accueil, espaces techniques et ateliers d'animation compris) dont $1700 \mathrm{~m}^{2}$ d'exposition pour les petits (augmentation de $110 \%$ environ) et $2200 \mathrm{~m}^{2}$ pour les grands (augmentation de $25 \%$ environ).

- renouveler l'offre en proposant de nouvelles activités, de nouveaux sujets, notamment pour satisfaire un public assidu. En effet, la moitié environ des visiteurs de la Cité des Enfants y sont déjà venus. Nous nous devions de leur proposer du nouveau. Ce renouvellement était également nécessaire pour remplacer certains jeux devenus démodés ou obsolètes.

- proposer une meilleure qualité de visite : espace moins dense, plus confortable en terme d'acoustique, de climatisation et plus lisible en termes de contenus. Le confort de visite est en effet un critère d'appréciation essentiel pour le public d'aujourd'hui devenu plus exigeant et plus familier avec les expositions. Une meilleure lisibilité des thèmes et des activités proposés est aussi une demande récurrente du public de la Cité des Sciences et de l'Industrie en général.

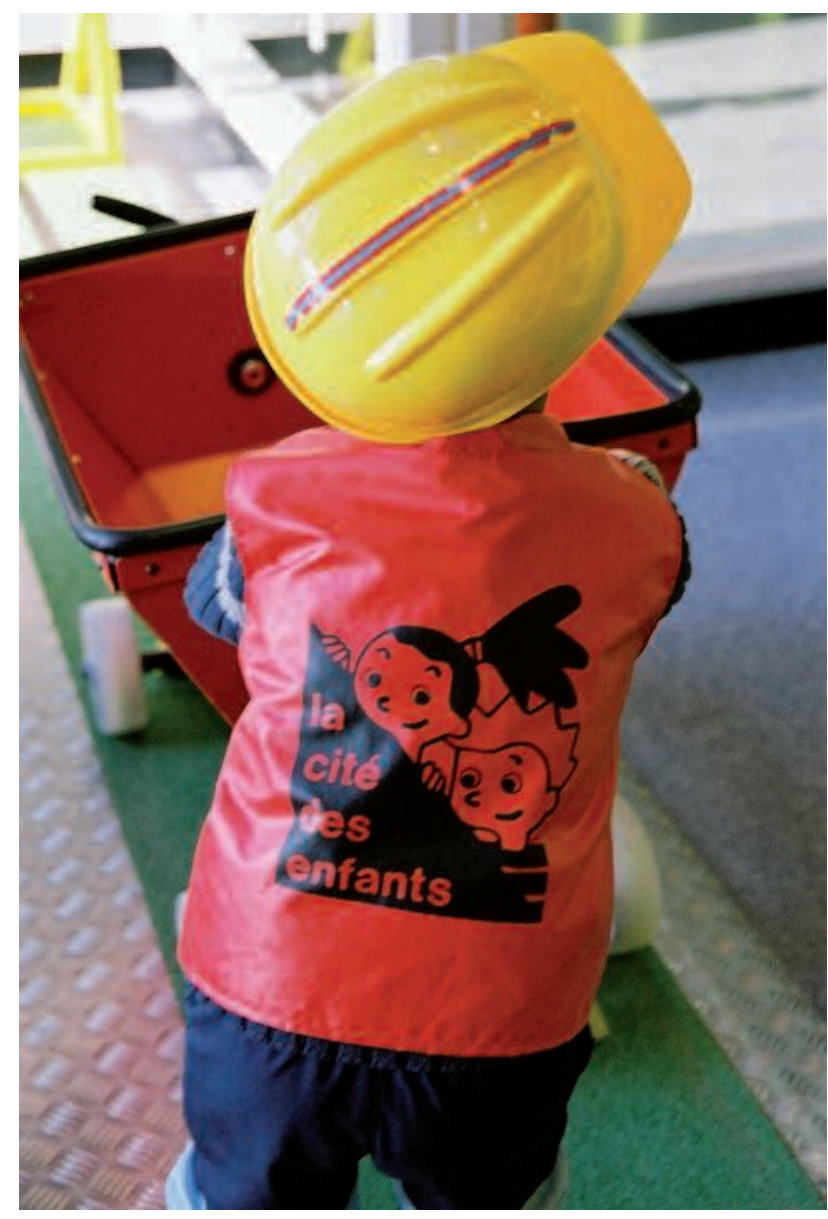

La Cité des Enfants d'origine reposait sur une muséologie favorisant l'action et l'interactivité. 


\section{Quelques valeurs sûres conservées}

Dans le cadre de ce renouvellement, nous avons néanmoins souhaité conserver les présentations les plus remarquables qui ont fait le succès de la Cité des Enfants d'origine pour satisfaire les enfants fidèles qui aiment à retrouver leurs jeux préférés : - pour la Cité des Enfants 2-7 ans, Le chantier, La machine à boules de Pierre Andrès, Le ruisseau, Se voir dans un miroir sont maintenus après rénovation. D'autres éléments, tels les jeux d'eau, sont repris sous d'autres formes.

- pour la Cité des Enfants 5-12 ans, La fourmilière, La serre à papillons, Fais la course avec ton squelette, Les machines à monter l'eau seront également maintenus.

\section{Une rénovation en deux temps}

Afin d'étendre dans le temps un projet d'une telle envergure et ne pas pénaliser le public par des fermetures d'espaces d'exposition trop longues, la rénovation de la Cité des Enfants s'opère en deux temps :

- décembre 2007, ouverture de la Cité des Enfants 27 ans, soit $2000 \mathrm{~m}^{2}$ d'exposition et d'équipements (ateliers pédagogiques, espace d'animations, locaux techniques) proposant une centaine d'éléments d'exposition pour les petits dont la grande majorité sont des créations. La Cité des Enfants 2-7 ans se déploie sur un espace entièrement réhabilité qui abritait anciennement des bureaux et des espaces de services. - avril 2009, ouverture de la Cité des Enfants 5-12 ans, soit $2400 \mathrm{~m}^{2}$ dont $2100 \mathrm{~m}^{2}$ d'exposition pour les plus grands. La nouvelle Cité des Enfants 5-12 ans se déploiera à l'emplacement de la Cité des Enfants d'origine et un certain nombre de présentations sont des reprises d'éléments existants.

Ainsi réorganisée, la nouvelle Cité des Enfants augmentera ses capacités d'accueil d'environ $40 \%$ par rapport à la Cité des Enfants d'origine. À chaque séance de 1 h 30, environ 1000 personnes pourront être simultanément présentes dans les deux espaces d'exposition. Cette capacité maximale pourra être atteinte lors des périodes de très fortes fréquentations aux vacances scolaires d'automne et de Noël.

\section{Notre public}

Le travail de réflexion qui a présidé à ce renouvellement a été l'occasion de nous interroger à nouveau sur la place des enfants dans des musées, notamment scientifiques tels que le nôtre. Concevoir un espace pour enfants nécessite avant tout de bien connaître leurs particularités, leurs intérêts, leurs capacités, bref, ce qu'ils sont. Cette connaissance de l'enfant s'est forgée sur notre propre expérience

\section{La Cité des Enfonts et les autres musées}

La Cité des Sciences et de l'Industrie fut l'un des musées pionniers en matière d'espace d'exposition pour enfants. Lors de sa création, en 1986, elle était l'un des premiers musées européens à proposer un espace de découvertes scientifiques dédié aux enfants. Aujourd'hui, la situation a changé car pratiquement tous les musées de sciences disposent d'un espace enfants ou en ont un en projet. Toutefois, l'originalité de la Cité des Sciences et de l'Industrie réside dans son offre pour les très jeunes enfants, à savoir pour les moins de 6 ans, les espaces enfants des musées étant généralement destinés aux enfants à partir de 6-7 ans. Cette particularité «française » fait écho à notre politique éducative pour les touts petits qui permet aux parents de scolariser leurs enfants dès 3 ans dans des écoles maternelles publiques. La démarche de la Cité des Enfants s’inscrit dans cet intérêt marqué pour l'apprentissage des très jeunes enfants.

basée sur l'observation de nos jeunes visiteurs depuis l'ouverture de la Cité des Enfants. Elle résulte aussi des échanges constants que nous avons entretenus tout au long de l'élaboration du projet avec des experts de l'enfance : pédagogues, psychologues, enseignants, sociologues...

Les petits...

Entre 2 et 7 ans, l'enfant connaît une période d'intense développement physique, intellectuel et affectif. Il a une certaine autonomie physique, parle, pose des questions, croit tout pouvoir faire, explore ou interprète à sa façon le monde qui l'entoure. Il vit une période riche en apprentissages spontanés. Mais sa pensée, dans sa forme infantile féconde, est encore immature et se façonne sous la double influence de la vie imaginaire et de l'expérience du réel. Encore très centré sur lui-même, le jeune enfant s'intéresse avant tout à ce qu'il sait faire. N'ayant pas encore atteint "l'âge de raison ", il ne peut pas comprendre objectivement le monde qui l'entoure et aborder des apprentissages complexes. Cette période de l'enfance est un moment structurant pendant lequel le jeune enfant construit son identité, grandit, prend de l'assurance. La vocation de la Cité des Enfants 2-7 ans n'est donc pas de lui transmettre des contenus, notamment scientifiques, 


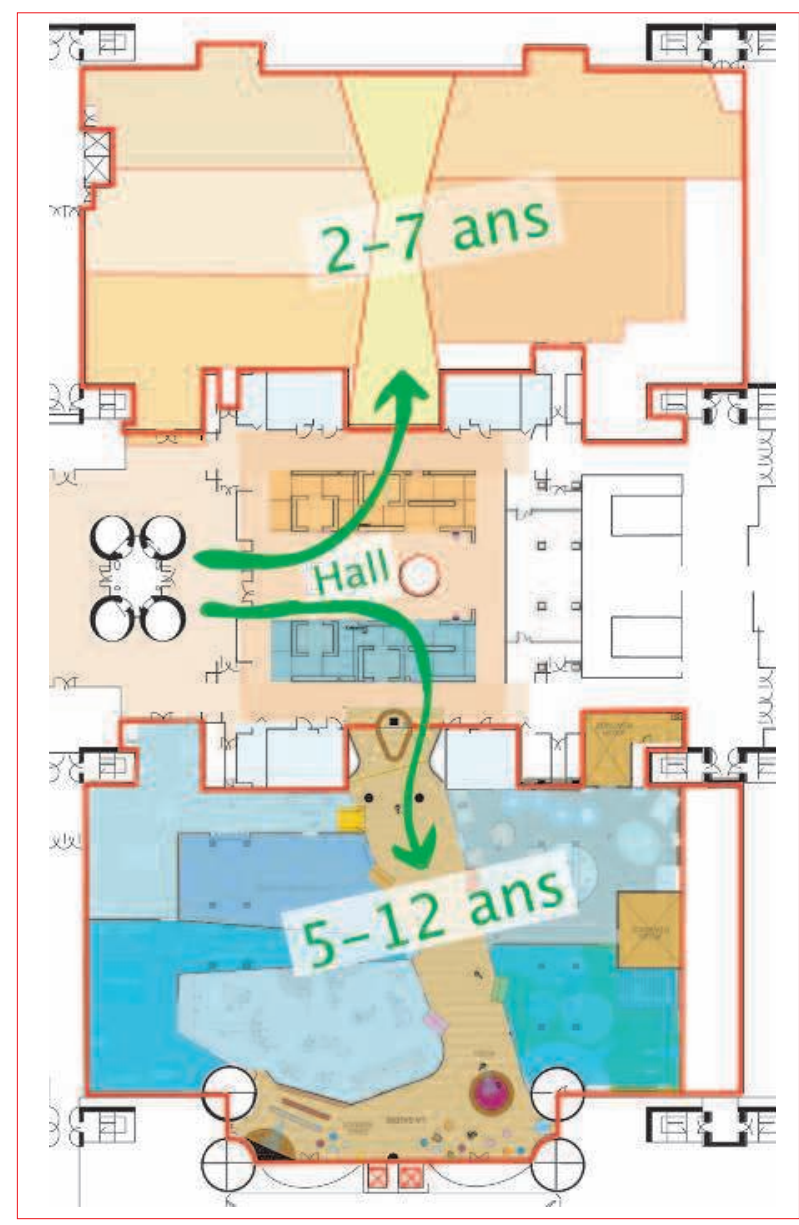

La Cité des Enfants se déploie maintenant sur toute une travée du niveau 0 .

(c) $\mathrm{CSI}$

mais bien de l'aider à se développer, de valoriser ses compétences, de consolider sa confiance pour contribuer à la réussite des futurs apprentissages qu'il pourra aborder plus tard quand il saura raisonner.

\section{... et les plus grands}

Après 6-8 ans, l'enfant a passé le cap de l'âge de raison et entre dans la « seconde enfance ». Il a maintenant une certaine capacité à s'extraire de son environnement immédiat pour raisonner. Il se défait d'une pensée magique pour s'essayer à des explications objectives. Observer un phénomène, tenter de le comprendre, voire de le conceptualiser, le relier à d'autres sont à sa portée. En pleine construction identitaire, il porte toujours un regard narcissique sur son corps et commence à se comparer aux autres. La vie sociale devient également essentielle, le meneur est celui qui a le plus d'idées, celui qui prend le plus d'initiatives. C'est la période des jeux dans la cour, des goûters d'anniversaires ; des groupes se forment auxquels on appartient ou pas.
L'enfant passe de l'égocentrisme à l'aptitude à se mettre à la place de l'autre dont il commence à saisir les intentions.

Compte tenu de la maturité physique et cognitive des plus grands, la Cité des Enfants 5-12 ans propose des activités ouvertes sur le monde et sur les autres. L'initiation à une culture scientifique et technique devient possible. Elle prend tout son sens pour des enfants capables de raisonner et devenus plus autonomes.

\section{L'âge charnière}

Cette distinction entre petits et grands n'est bien évidemment pas une frontière rigide. Entre 5 et 7 ans, certains enfants encore petits aspirent à devenir grands tandis que d'autres, déjà grands, aiment à rester petits... La nouvelle Cité des Enfants tient compte de cet âge charnière en créant une superposition de cette tranche d'âge entre les deux expositions. Les enfants de 5 à 7 ans auront donc la possibilité de visiter l'une ou l'autre des expositions, selon leur niveau de développement et d'autonomie, leurs centres d'intérêts, leur fratrie, voire leur humeur du jour...

\section{Les grands axes de la rénovation}

Notre connaissance du public enfant ainsi que les études d'évaluation sur les pratiques de nos visiteurs nous ont aidés à bâtir les points forts de la nouvelle Cité des Enfants. Des études sont en effet régulièrement pratiquées par notre département «Évaluation et Prospective » qui mène des enquêtes quantitatives (fréquentation des expositions) et qualitatives (satisfaction du public) auprès de nos visiteurs, au cours de leur visite ou à la sortie des expositions. Ces études font l'objet de rapports internes et de quelques publications.

Les animateurs de la Cité des Enfants, qui reçoivent le public et organisent des animations à toutes les séances, recueillent également les avis spontanés des visiteurs.

\section{Un public plus jeune}

Des enquêtes faites à la Cité ou dans d'autres lieux culturels montrent que les parents et enseignants sont fortement demandeurs d'une offre culturelle éducative pour de très jeunes enfants hors du cadre scolaire. C'est pourquoi nous avons décidé de nous adresser à un public plus jeune qu'auparavant, soit aux enfants dès 2 ans. Mais la grande différence d'aptitude physique et cognitive entre un enfant de 2 ans et de 7 ans ne permet pas de concevoir les mêmes jeux pour tous. Les activités de la Cité des 


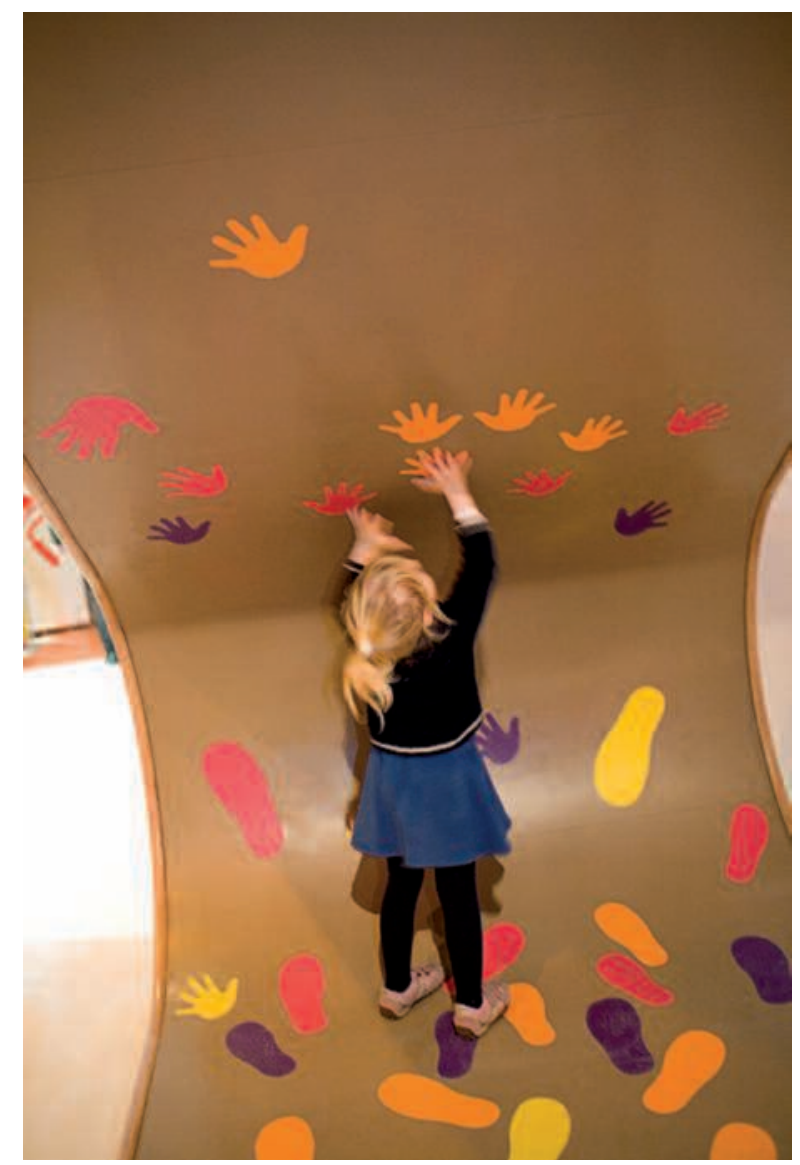

Le design des activités conçu par l'agence Du\&Ma favorise une appropriation intuitive par les enfants. (C) CSI/Sophie Chivet

Enfants 2-7 ans sont donc majoritairement conçues pour des enfants de 3 à 5 ans (tranche d'âge plus homogène), alors que certaines sont spécifiquement dédiées aux plus jeunes (2-3 ans) et d'autres, plus complexes, sont profitables aux grands de 6-7 ans.

\section{Des contenus renouvelés}

La Cité des Enfants 2-7 ans aborde des sujets qui n'étaient pas présents dans l'ancienne Cité des Enfants comme l'émotion et l'identité sociale. Il est en effet largement reconnu par les professionnels de l'enfance que les expériences émotives et l'environnement social du jeune enfant sont indispensables à la construction de sa personnalité, donc à sa capacité à apprendre. D'autres nouveautés sur l'interprétation des images (la découverte indicielle de photographies, par exemple, ou encore la construction d'une histoire à partir d'images), ou bien sur l'écriture (tracer avec un crayon lumineux, associer des graphèmes à des phonèmes) font maintenant partie de l'offre. Bien d'autres nouveautés sont proposées.
Dans la future Cité des Enfants 5-12 ans, une nouvelle thématique, Communiquer, abordera l'importance de l'échange et du langage chez les enfants, dont le rôle est fondamental dans la construction de sa pensée. D'autres sujets, tels que les énergies renouvelables, l'astronomie ou les techniques vidéo feront leur entrée dans l'exposition ou seront renforcés par rapport à la Cité des Enfants d'origine.

\section{Une exposition mieux structurée}

Contrairement à l'ancienne Cité des Enfants, les éléments d'exposition de la Cité des Enfants 2-7 ans ne sont pas disposés sur un vaste espace unique mais répartis dans 5 espaces de 200 à $300 \mathrm{~m}^{2}$ chacun. Le jeune enfant est en effet très sensible à l'agitation de son entourage, de sorte qu'il est facilement dissipé par ce qui se passe autour de lui, ce qui peut nuire à sa visite. Cette structuration en petits espaces limite donc la dispersion de l'enfant et permet aux parents d'avoir toujours leurs jeunes enfants à portée de vue. Ces espaces sont distribués autour d'une large artère centrale. Chacun correspond à une thématique qui, compte tenu de la spécificité des jeunes enfants évoquée plus haut, se réfère aux capacités de l'enfant et non aux contenus proposés dans les activités. Ainsi, les titres de ces espaces utilisent massivement le « je » :

- Je me découvre, où l'enfant prend conscience de son corps, de ses émotions et de ses capacités sensorielles; - Je sais faire, où il exerce ses facultés cognitives (classer, anticiper, associer...) ;

- Je me repère, où il apprend à se repérer dans l'espace et le représenter;

- Tous ensemble, où il peut s'associer à d'autres pour faire des activités à plusieurs ;

- J'expérimente, où il fait des expériences avec des éléments familiers tels que l'eau, l'air ou la lumière. Ces éléments ne sont pas mis en perspective avec des problématiques scientifiques. Ce sont juste des matières que l'enfant peut manipuler pour aiguiser sa curiosité : si je fais ceci, il se passe cela, si je recommence, c'est pareil, si je fais différemment, il se passe autre chose. Le plaisir de faire des expériences sans forcément comprendre ce qui se passe est un passage obligé pour s'intéresser plus tard aux sciences et techniques. La curiosité est le fondement de la démarche scientifique.

La rénovation de l'exposition pour les 5-12 ans s'inscrit dans cette même logique d'organisation spatiale autour d'une artère centrale. En revanche, compte tenu de la maturité des plus grands enfants, les thématiques de l'exposition seront cette fois qualifiées par leurs contenus: Le corps, Communiquer, 


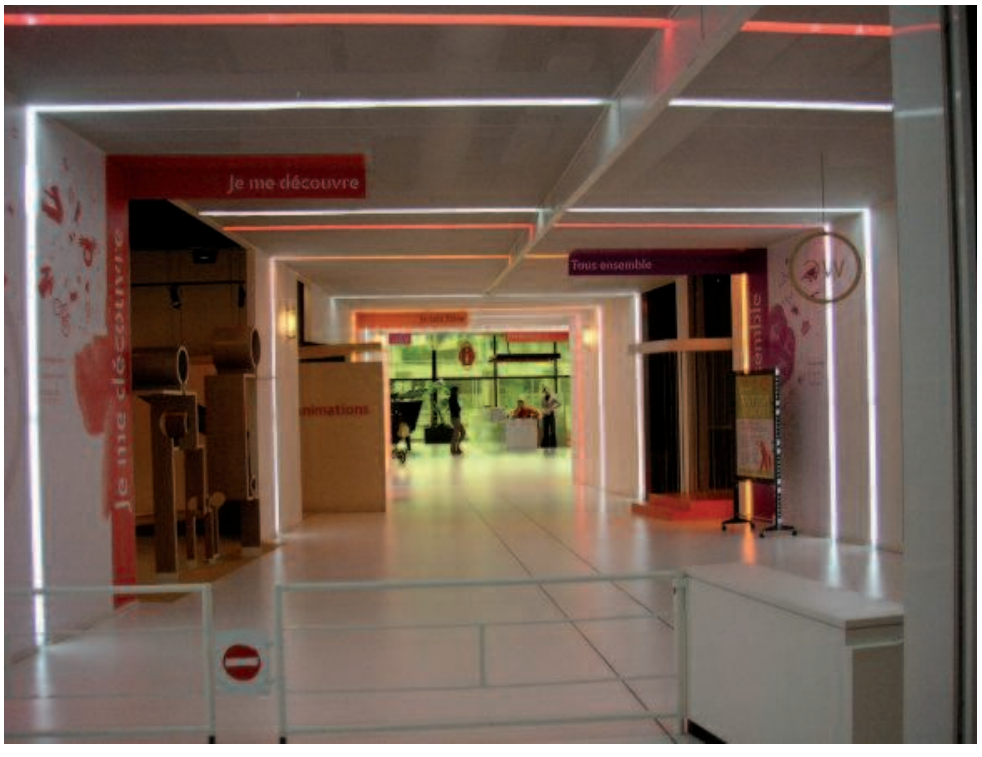

La scénographie conçue par Arnaud Sompairac distribue les espaces thématiques autour d'une artère centrale et qualifie clairement chaque espace.

(c) CSI

Le jardin, Le studio TV, L'usine, Les jeux d'eau. Les thèmes centrés sur l'enfant et ses relations aux autres, Le corps et Communiquer, seront moins nombreux que les quatre autres ouverts sur le monde.

\section{Une muséologie ancrée dans le réel}

Comme il y a 15 ans, nous souhaitons faire de la Cité des Enfants une exposition de référence, un lieu qui propose une expérience unique que l'enfant n'a pas l'occasion de vivre ailleurs, à la maison ou à l'école. Certains noteront qu'il y a peu d'écrans dans cette nouvelle Cité des Enfants... C'est un choix délibéré car la plupart des enfants d'aujourd'hui, même très jeunes, utilisent fréquemment un ordinateur chez eux ou dans leur classe. D'où notre volonté de nous démarquer et d'ancrer les activités dans le réel où l'implication sensitivo-motrice de l'enfant domine. Ce parti pris muséologique a d'autant plus de sens que le développement cognitif et le processus de mémorisation chez l'enfant sont intimement liés à ses activités corporelles, particulièrement pour les jeunes enfants. Ils aiment avant tout bouger, sauter, courir et enregistrent ainsi, plus ou moins consciemment, des informations au cours de jeux qui engagent leur corps.

Un élément d'exposition illustre tout particulièrement ce principe de réalité. Il s'agit d'un jeu où il faut associer des animaux à leur peau, leur nombre de pattes, leur empreinte ou leur petit. Ce type de jeu existe souvent sous forme de jeux de cartes, voire de jeu multimédia, où l'association se fait par l'image uniquement. À la Cité des Enfants, l'enfant va utiliser de vrais objets (des moulages d'animaux) : il prend un animal dans un bac de rangement puis va le poser sur des tablettes où figurent les différentes solutions. Si l'association est juste, une lumière verte s'allume et un «bravo » se fait entendre. La relation physique qui se joue ici entre l'enfant et le jeu prendre un objet, se déplacer, le poser... recommencer - renforce le process de mémorisation des associations qu'il a effectuées par rapport à un jeu purement mental sur table ou sur écran.

Par ailleurs, tous les éléments d'exposition de la nouvelle Cité des Enfants ont été conçus pour favoriser au mieux une appropriation intuitive et immédiate de l'activité par l'enfant. Le design des éléments s'affranchit du superflu : peu de mobilier ou d'interface, la forme induit le geste ou la posture. Pour les mêmes raisons, la scénographie est volontairement épurée : chaque espace thématique a sa propre identité qualifiée par ses matériaux, ses couleurs, ses lumières mais ne contient pas d'objets contextualisant - décors, maquettes, illustrations, photos...en relation avec la thématique. Seules se distinguent les activités pour les enfants.

Un autre principe muséologique fort de la nouvelle Cité des Enfants est de favoriser des activités qui se font à plusieurs. La encore, nous nous démarquons de l'univers familier de l'enfant en lui proposant des jeux de grande dimension qui impliquent plusieurs participants. La muséologie collective encourage également l'imitation entre enfants qui est un ressort essentiel de l'apprentissage.

\section{Un travail d'équipe}

Un projet de cette envergure mobilise toute une équipe de concepteur/producteur d'exposition. L'équipe de la Cité des Sciences et de l'Industrie s'est entourée pour cela de collaborations externes indispensables à la réussite du projet.

D'une part, la conception du programme conduit par Orna Cohen, muséographe, s'est fait avec l'appui d'experts de la petite enfance (psychologues, éducateurs, sociologues...), en particulier avec Marie-Luce Gibello-Verdier, psychothérapeute d'enfants.

D'autre part, l'expression formelle de ce programme d'exposition est l'œuvre de l'agence Du\&Ma (pour le design de la plupart des éléments d'exposition), de l'agence Asa, Arnaud Sompairac (pour l'architecture et la scénographie des espaces) et de Gérard Plénacoste et Cécile Boyer (pour le graphisme de l'exposition). 


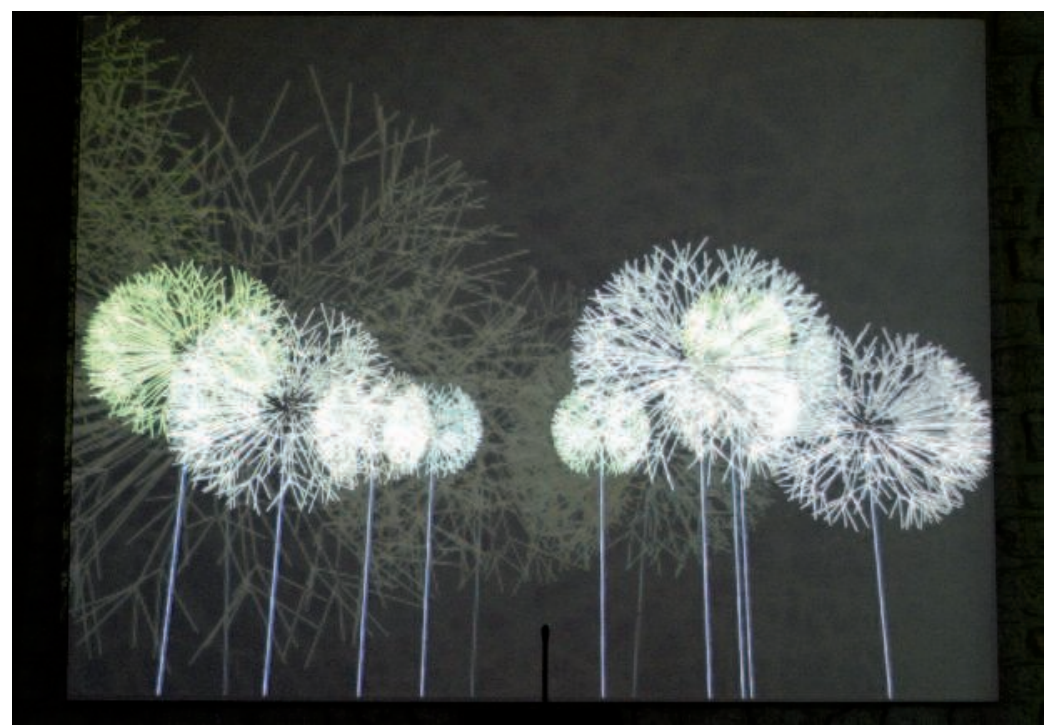

L'installation numérique Les pissenlits de Edmond Couchot et Michel Bret a été adaptée pour la Cité des Enfants 2-7ans. ๑) Numeriscausa/CSI

Un graphisme imagé

L'enfant de 2 à 7 ans n'est pas lecteur. Aussi toute aide ou information écrite à son attention est-elle inadaptée. Le graphisme de l'exposition pour les 27 ans fait donc la part belle à l'image : chaque élément est accompagné d'une consigne placée à hauteur d'enfant qui utilise des photographies d'enfants et des dessins pour signifier ce qu'il faut faire. Elle est aussi un outil pour l'accompagnateur qui peut lire à l'enfant la phrase de consigne. Cette communication repose donc sur une identification de l'enfant-visiteur à l'enfant-démonstrateur : un enfant montre à un enfant ce qu'il faut faire. Il en va de même pour la dénomination des espaces thématiques. Bien que destinés surtout aux adultes, leur titre est enrichi d'une photographie d'enfants qui illustre la thématique.

Ce principe graphique sera repris pour la future Cité des Enfants 5-12 ans dans laquelle l'écrit sera, en plus, un appui pédagogique utilisé pour donner des explications et contextualiser les jeux. Toutefois, cet usage restera limité à de très courts textes tant l'enfant, même lecteur, est peu enclin à lire dans une exposition. D'autres lieux, comme l'école ou la maison, sont plus adaptés à une pratique de lecture attentive.

Une sensibilisation aux œuvres artistiques

La nouvelle Cité des Enfants s'intéresse également à l'éveil artistique et à l'ouverture sur l'imaginaire des enfants. La Cité des Enfants 2-7 ans fait ainsi appel à des artistes, tels que Pierre Andrès, Edmond Couchot ou Jean-Pierre Larroche pour concevoir certains éléments. Les enfants sont proches de cette forme de sensibilité et les réalisations d'artistes stimulent leur pensée. La présence d'œuvres renforce par ailleurs la dimension esthétique de l'exposition.
Une visite plus agréable

Au-delà de l'intérêt ou des critiques que nos visiteurs portent aux contenus de nos expositions, leurs remarques sur les conditions de visites sont fréquentes. Les tracas concernant l'accueil, le confort de visite, l'accès aux services du musée (sanitaires, vestiaires...) font partie des exigences récurrentes qui se dégagent de nos études d'évaluation. La nouvelle Cité des Enfants propose donc une amélioration des services offerts aux visiteurs : hall d'accueil plus spacieux et plus convivial, sanitaires équipés pour les tout petits, ainsi qu'un meilleur confort de visite des espaces d'expositions : climatisation rénovée, acoustique renforcée, sièges pour les accompagnateurs...

\section{La place de l'adulte}

Comme évoqué plus haut, nous avons toujours tenu compte du rôle que peut tenir l'adulte pendant la visite des expositions pour enfants, autre que celui de porter les manteaux... Donner une véritable place à l'accompagnateur était motivé par de nombreuses recherches montrant à quel point la coéducation était un véritable levier dans le désir d'apprendre. La Cité des Enfants est un lieu propice pour partager un moment d'échange et de plaisir entre enfants et adultes. C'est pourquoi, les enfants doivent être accompagnés d'un adulte pendant toute la durée de la visite.

\section{Jover un rôle pendant la visite}

L'une des innovations de la nouvelle Cité des Enfants 2-7 ans est de proposer explicitement à l'adulte un rôle à tenir pendant la durée de sa visite par l'intermédiaire d'un texte à l'entrée de chaque espace. Ce rôle peut être induit par les scénarios de 
jeux ou par l'agencement muséographique : hublot pour encourager, sièges pour tenir à distance... Nous avons identifié trois rôles essentiels que peut prendre l'adulte accompagnateur :

- l'adulte observateur : il est à l'extérieur du jeu mais peut voir jouer son enfant pour le rassurer par sa présence. Cette attitude est adaptée aux activités collectives, comme Le chantier, où plusieurs enfants se côtoient. Ce moment d'observation permet à l'adulte de voir son enfant agir, faire, mais aussi interagir avec d'autres enfants ;

- l'adulte supporteur : il adopte ici une attitude encourageante qui stimule, soutient l'enfant dans l'épreuve. Il se tient à distance de l'enfant pour le laisser faire seul mais reste suffisamment proche pour le rassurer. Ce regard bienveillant

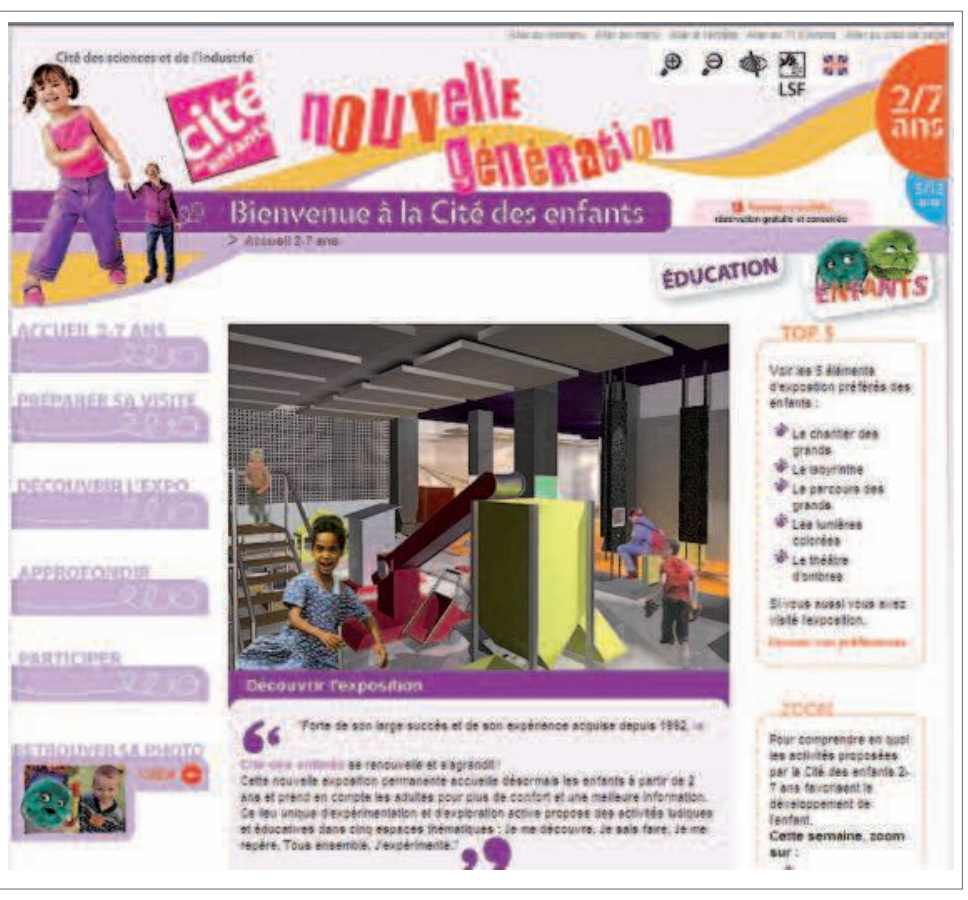

Le site Internet de la Cité des Enfants permet tout autant de préparer sa visite que la poursuivre chez soi. () CSI

- l'adulte collaborateur : il fait avec l'enfant. Il se prête au jeu, sans pour autant oublier son rôle d'adulte. Ici, le dispositif muséographique doit indiquer clairement à l'adulte qu'il peut participer à l'activité, qu'il est invité à toucher, à tourner la manivelle, à mesurer sa taille... bref, à faire avec ou à se comparer à son enfant.

\section{Prolonger sa visite}

Parce que l'adulte est trop occupé à observer, encourager ou jouer avec son enfant, ou simplement à ne pas le perdre de vue... nous savons qu'il est peu disponible pendant la visite pour s'informer sur les tenants et les aboutissants des situations de jeux proposés. Aussi, avons-nous limité l'information destinée aux adultes dans l'exposition à de courts textes de présentation dans chaque espace thématique. En revanche, nous l'incitons à prolonger sa visite chez lui sur le site de la Cité où il pourra trouver des descriptions et des éclairages psycho-pédagogiques sur les activités de l'exposition. Pour cela, chaque espace thématique contient un renvoi signalétique vers le site www.citesciences.fr. Ce site est également un outil de préparation à la visite où on y trouve des informations pratiques, une description de l'exposition et des propositions de parcours de visites.

Un nouveau guide de la Cité des Enfants 2-7 ans a également été édité à l'occasion de cette rénovation et propose une description exhaustive des activités de l'exposition.

\section{L'offre d'animations}

La médiation fait partie intégrante de la Cité des Enfants depuis sa création. La présence d'animateurs dans les expositions est assurée en permanence. À chaque début de séance, des animateurs accueillent les visiteurs pour les guider et les conseiller dans leur visite. Un animateur assure par ailleurs une permanence au « Point information » de chaque exposition pour répondre aux demandes des visiteurs pendant toute la séance. Et enfin, divers types d'animation sont proposés à chaque séance : - des animations "éclairs » à proximité de certains éléments permettent d'approfondir leurs contenus ou mieux comprendre l'activité elle-même ;

- des ateliers d'éveil, pour les petits, ou de découvertes scientifiques, pour les grands, sont proposés à des petits groupes, en public individuel ou scolaire, dans les ateliers pédagogiques situés dans chacune des expositions ; - des animations-spectacles de 20 minutes sont proposées aux familles au cours de chaque séance des vacances scolaires; ces animations de grande capacité d'accueil se déploient dans l'espace animations de chaque exposition qui peut accueillir une centaine de visiteurs.

Par ailleurs, des animations spécifiques pour des groupes scolaires sont organisées avec les écoles tout au long de l'année. 


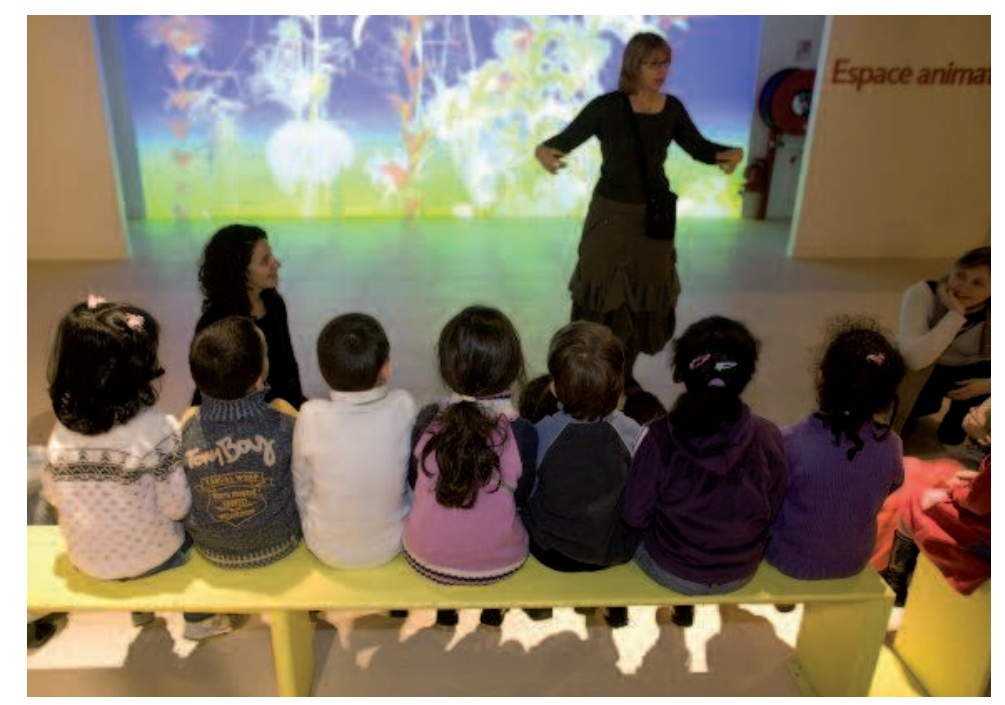

Animation proposée aux jeunes enfants lors d'une visite. (c) CSI/Sophie Chivet

La rénovation de la Cité des Enfants a été conçue et menée dans l'esprit de la Cité des Enfants d'origine : offrir aux jeunes enfants l'occasion de vivre des situations d'exploration variées, plaisantes et intéressantes qui ne peuvent être vécues ailleurs dans le cadre familial ou scolaire. À travers les activités proposées, notre objectif est de contribuer au développement de l'enfant et à aiguiser sa curiosité, laquelle est un tremplin pour de futures vocations scientifiques. Nous cherchons toujours pour cela à nous enrichir des expériences des professionnels de l'enfance et des travaux des chercheurs qui mettent en valeur l'enfant et le prennent au sérieux. Car l'enfant n'est pas un visiteur d'expositions simplement plus jeune que les autres. Il n'est pas un «petit adulte». Son approche du monde se fait essentiellement à travers des expériences physiques et affectives. Aussi la Cité des Enfants ne cherche-t-elle pas à en faire un petit savant ni même un petit scientifique. Elle ambitionne plutôt de lui proposer des activités qui favorisent une relation corporelle, sensorielle ou émotive à des contenus et qui contribuent ainsi à son initiation aux sciences et aux techniques. La mémoire silencieuse de son corps enregistre les informations glanées au cours des jeux. Gageons que ces « empreintes » de visite lui seront utiles plus tard pour s'adapter à des situations inconnues et serviront de trame inconsciente aux apprentissages qu'il abordera quand il sera plus grand ou dans d'autres lieux. Gageons également que la visite à la Cité des Enfants marquera sa mémoire, lui donnera envie d'y revenir, voire d'y amener ses propres enfants dans une vingtaine d'année... 